\title{
TUTORÍA ENTRE PARES: EXPERIENCIA DEL PROGRAMA ASESORÍA PEDAGÓGICA ESTUDIANTIL DE LA UNIVERSIDAD CATÓLICA DE SANTIAGO \\ DE GUAYAQUIL
}

\author{
COUPLES TUTORING: UNIVERSIDAD CATOLICA SANTIAGO DE GUAYAQUIL \\ PEDAGOGICAL STUDENT COUNSELING PROGRAM EXPERIENCE
}

\section{YADIRA BLAKMAN BRIONES', ELBA BERMÚDEZ REYES ${ }^{2}$}

1 Universidad Católica de Santiago de Guayaquil. yadira_blakman@hotmail.com

2 Universidad Católica de Santiago de Guayaquil. elba.bermudez@cu.ucsg.edu.ec

Una de las mayores preocupaciones de las instituciones de educación superior, es la deserción y el bajo rendimiento académico que afecta tanto a las universidades como a los estudiantes. Ante esta realidad, surge la necesidad de incorporar nuevas estrategias pedagógicas que contribuyan a reducir el impacto de este problema. En la Universidad Católica de Santiago de Guayaquil, se implementó la asesoría pedagógica estudiantil (APE) a través de la modalidad tutoría de pares, que consiste en el acompañamiento, orientación y apoyo, brindado por estudiantes de alto rendimiento académico a sus pares para que puedan mejorar su desempeño académico. Los objetivos de este trabajo de investigación, fueron: Determinar el nivel de efectividad del programa Asesoría Pedagógica Estudiantil en las nueve Facultades de la Universidad Católica de Santiago de Guayaquil (UCSG), evaluar el desempeño académico de los estudiantes bajo muestra e identificar los factores que les dificultan el aprendizaje, se consideró importante mejorar significativamente el programa. Se trata de un estudio cuantitativo, descriptivo y transversal, en el que participaron 312 estudiantes tutorados por el programa y 58 docentes. Como resultado, se obtuvo el nivel de efectividad del programa y de qué manera este contribuyó a mejorar el nivel académico.

PALABRAS CLAVE: Acompañamiento Académico, Tutoría entre pares, Asesoría pedagógica, Estudiante Tutor. Counseling, Student Tutor.

ABSTRACT

One of the biggest concerns of higher education institutions is the desertion and low academic performance that affects universities and students. Faced with this reality, there is a need to incorporate new pedagogical strategies that contribute to reducing the impact of this problem. At Universidad Católica de Santiago de Guayaquil, student pedagogical counseling (APE) was implemented through the couples tutoring modality, that consist in an accompaniment, guidance and support provided by students of high academic performance to their couples so they can improve their academic performance.The objectives of this research were: To determine the effectiveness level of the Student Pedagogical Counseling Program in the nine Faculties of the UCSG, to evaluate the academic performance of the students under sample and to identify the factors that made difficult their learning, it was considered significantly important to improve the program. It is a quantitative, descriptive and cross-sectional study, in which participated 312 students tutored by the program and 58 teachers. As a result, the effectiveness level of the program was obtained and the way it contributed to improve the academic level.

KEYwords: Academic Accompaniment, Couples Tutoring, Pedagogical 


\section{INTRODUCCIÓN}

En los últimos años la educación superior está sufriendo grandes transformaciones que están relacionadas con la pertinencia y la calidad de la educación superior. Las últimas reformas universitarias planteadas por la UNESCO (1998), se encaminan hacia el desarrollo de la máxima implicación de los estudiantes en su propio aprendizaje y la adquisición de competencias que les permitan formarse y poder aprender a lo largo de la vida. El aprendizaje centrado en el estudiante, planteado por las últimas reformas universitarias, recomienda crear ambientes de aprendizaje dónde los estudiantes tengan altos niveles de participación, autonomía y ser protagonistas de su propio proceso de aprendizaje. En esa misma línea de pensamiento Lobato y Nagore (2016, p.379) agregaron "como sujeto activo que aprende autónomamente competencias personales y profesionales".

Este mismo planteamiento de centralidad del estudiante ha sido recogido por el modelo educativo pedagógico de la Universidad Católica de Santiago de Guayaquil, sin soslayar el importante rol que juega el docente como facilitador, mediador en el proceso de aprendizaje.

El ingreso a la universidad para un estudiante, implica cambios en su proceso de desarrollo psicosocial; la vida universitaria en muchos casos significa dejar la zona de confort para asumir nuevas responsabilidades, entre ellas, la autonomía sobre su reciente etapa y todo el proceso de desarrollo que esto conlleva, algunos deben separarse de sus familias o residir en una nueva ciudad, establecer nuevas relaciones interpersonales o tomar decisiones importantes acerca de su proyecto de vida profesional y personal, si este paso no se realiza en forma adecuada, podrían derivar en crisis, que afectarán sus actividades sociales y académicas, provocando en situaciones más graves el abandono de los estudios.

El bajo rendimiento académico, la deserción y el rezago, es otra de las dificultades que afrontan las universidades del país, situación que también se evidencia en estos últimos años en la UCSG, principalmente con los estudiantes de los primeros años, afectando la eficiencia terminal de las carreras. En este contexto, la problemática no solo preocupa a las autoridades educativas del sistema de educación superior, sino también a las autoridades gubernamentales que han tenido que generar políticas públicas para elevar la calidad de la educación y lograr los resultados esperados.
Para Bianculli y Marchal (2013, p. 381) esta situación se ha convertido en un "obstáculo y un problema estructural, que está dando origen a la emergencia de políticas educativas vinculadas a favorecer el ingreso, la retención y la graduación de los alumnos". Por lo tanto institucionalizar un programa de tutorías entre pares, de acuerdo con la experiencia de otras universidades y la nuestra, favorecerá el desarrollo integral del estudiante, al ser una estrategia metodológica educativa, mejorará el rendimiento académico y la calidad de la educación.

En la tutoría entre pares, el docente es el guía para el estudiante-tutor, a quién necesita afianzar y fortalecer en su papel; requiere que el profesor lo apoye, lo respalde, lo oriente, de manera que le permita acceder al conocimiento fundamental y a las herramientas tutoriales necesarias que tendrá que desplegar a lo largo de esta labor pedagógica.

Su aporte, servirá para disminuir el rezago y el abandono educativo en las aulas universitarias; favorecerá su desarrollo psicosocial y mejorará la calidad de la educación superior, de ahí se deriva la importancia de conocer los factores que influyen en el desempeño académico para adecuar nuestro programa de apoyo pedagógico estudiantil APE, de acuerdo a las necesidades de los estudiantes y las exigencias académicas.

Como precedente, se han encontrado varios estudios internacionales sobre asesoría pedagógica y tutoría universitaria, generalmente encausados al acompañamiento del docente hacia el estudiante. En el caso particular de Ecuador, si bien es cierto es un tema de interés, hay muy pocas experiencias investigativas sobre la tutoría entre pares en los espacios universitarios.

Investigaciones previas dan cuenta de la complejidad del sistema de educación superior, de los procesos de enseñanza-aprendizaje centrado en el estudiante y de las nuevas exigencias para convertirlo en un ser crítico, reflexivo, propositivo y autónomo. Algunos estudios han indicado que las actividades colaborativas entre compañeros mejoran el rendimiento académico, en el cual influyen varios factores, entre los que se mencionan están las estrategias de aprendizaje de los estudiantes, la metodología de enseñanza del docente, el entorno familiar, la motivación y el ambiente que rodea al estudiante.

Por otra parte, se ha señalado que la tutoría entre pares aporta al desarrollo académico integral, pues se potencian las habilidades 
cognitivas, sociales y afectivas, necesarias para el desempeño académico, el aprendizaje autónomo, la autoestima y el desarrollo de la comunicación como competencia social básica. También se ha encontrado que trabajando en forma colaborativa, la ayuda ofrecida entre compañeros, desarrolla las capacidades para construir un conocimiento valioso al elaborar sus propios significados, de la misma forma proporciona confianza y autonomía en la autorregulación de los procesos.

Otros autores mencionan que favorece la integración de los estudiantes a la vida universitaria, la utilidad de proporcionarles información y orientación sobre asuntos académicos, administrativos y socio-afectivos.

\section{LA TUTORÍA UNIVERSITARIA}

La tutoría universitaria según Álvarez, (2014) es una función de acompañamiento, de orientación y de apoyo al estudiante, en su proceso de personalización de los aprendizajes y del desarrollo de las competencias, tanto a nivel personal como profesional; adopta diferentes modelos dependiendo de los contextos, la cultura universitaria, de las políticas y normas educativas, recursos disponibles y necesidades de los estudiantes.

Durán (2009) citando a Cannon-Bowers y Salas, (1997), menciona que en este contexto el desarrollo de las competencias individuales, toma protagonismo la competencia cooperativa, entendida como un conjunto de conocimientos, habilidades y actitudes necesarias para un trabajo en equipo efectivo. De esta manera, la cooperación se constituye como una competencia fundamental en la sociedad del conocimiento que facilita, no solo el desarrollo de habilidades y actitudes autónomas; sino que constituye un motor de aprendizaje que utiliza pedagógicamente las diferencias entre los estudiantes como un elemento a favor del aprendizaje.

La formación centrada en el estudiante y la adopción de una mayor calidad en la educación superior, según Durán y Flores (2014) conducen al enorme esfuerzo de implicación de los estudiantes, al buen conocimiento de sus potencialidades para que a partir de ahí poder gestionar gradual y apropiadamente su propio aprendizaje y el desarrollo de competencias que les permitan formarse y poder aprender a lo largo de la vida. Así mismo planteó que la competencia cooperativa que se pone en práctica en el contexto educativo, posteriormente se trasladan a contextos profesionales, donde el desempeño del trabajo en equipo es considerado esencial.

TUTORÍA ENTRE PARES EN LA UNIVERSIDAD

Durante la última década, en América Latina, el aprendizaje entre estudiantes, ha tomado un papel importante, se define a la tutoría entre pares como la ayuda, orientación o guía en determinados aspectos de la orientación de los aprendizajes y de la integración en la vida universitaria, prestada y desarrollada por estudiantes de un curso superior a un grupo pequeño de compañeros de los primeros años, según Albanaes et.al (2015), la responsabilidad y la coordinación de estos tutores estudiantes recae en un docente tutor.

En el ámbito de la educación superior, Durán y Flores, (2014); Albanaes et al. (2015), mencionaron que los beneficios de las tutorías de pares son diversos, entre ellos, se distingue su aporte pedagógico y social, así como el mejoramiento de la calidad de la experiencia universitaria, al crearse vínculos y fortalecer relaciones entre compañeros, ayudando al desarrollo social y cognitivo. En esa misma línea de pensamiento, los estudios de Sánchez Ávila, Macías, Almendra y Jiménez Leube, (2007), indican que la tutoría de pares, desde el punto de vista de la educación centrada en el estudiante es beneficiosa tanto para tutores como para tutorados en virtud que el tutor estudiante refuerza sus conocimientos y mejora sus habilidades sociales y pedagógicas. En cuanto a la relación con el tutorado, genera compromiso con el otro, acercándose a la realidad del estudiante hasta alcanzar juntos los objetivos propuestos (Casado-Muñoz et al., 2015).

\section{MATERIALES Y MÉTODOS}

Se trata de un estudio descriptivo, transversal, de enfoque cuantitativo, con análisis cualitativo, los resultados obtenidos en la investigación fueron recogidos en cuadros estadísticos; esta articulación de los datos, ofrecieron mejores posibilidades de estudio, análisis y reflexión.

Con respecto a los métodos y las técnicas de recolección de datos, se realizaron encuestas a los estudiantes y docentes; se implementó un muestreo no probabilístico intencional, puesto que los participantes eran estudiantes de primero a tercer ciclo, atendidos por el programa APE en las nueve facultades de la Universidad Católica de Santiago de Guayaquil, durante el período 2015 - 2016 y docentes de las asignaturas que constituyeron el nudo crítico de tipo 
académico. La población ascendió a un total de 312 estudiantes y 58 docentes.

La primera fase del proyecto consistió en medir la influencia del programa y tuvo como objetivo determinar el nivel de efectividad de la Asesoría Pedagógica Estudiantil (APE), en las diferentes Facultades de la UCSG, realizando una evaluación de los logros académicos de los estudiantes asesorados.

La muestra bajo estudio fue del $58 \%$ del universo de estudiantes atendidos por APE, se seleccionó intencionalmente a los estudiantes, en primera, segunda y tercera matrícula, con calificaciones inferiores a 6,5 puntos, que fueron derivados y atendidos por el programa por ser considerados en riesgo académico. El $42 \%$ de estudiantes que también fueron atendidos por el programa, no fueron seleccionados porque tienen buen rendimiento y asisten a los talleres para mantener sus promedios de calificaciones y becas.

Se creó una base de datos, con la información de los estudiantes en riesgo académico, derivados y atendidos por APE, matriculados en las nueve facultades que oferta la UCSG, en la que se determinaron: las asignaturas nudos críticos, las notas o calificaciones obtenidas durante los semestres A y B - 2015 y A y B - 2016, número de matrícula, número de estudiantes atendidos y número de estudiantes que mejoraron sus notas y aprobaron las materias. Estos datos fueron ordenados y registrados por facultades, carreras y semestres. Con los datos estudiados se procedió a su análisis cuantitativo y con ello obtener el nivel de efectividad de la ejecución del programa.

De toda la información ordenada y racionalizada en la base de datos, se obtuvo un cuadro estadístico, que refleja el porcentaje de efectividad logrado por el programa durante el período mencionado; para esto fue necesario la elaboración y aplicación de una escala tipo Likert y se determinaron tres baremos: Muy Satisfactorio, Satisfactorio y Poco Satisfactorio. Se determinó una escala numérica de medición del $10 \%$ al $100 \%$ como indicador del grado de intensidad de la eficiencia del programa, en el primer baremo Muy Satisfactorio, se consideraron los máximos puntajes de la escala, comprendidos entre $90 \%$ y $100 \%$, en el segundo baremo Satisfactorio, se consideraron los puntajes medios comprendidos entre el $50 \%$ y el $80 \%$ y finalmente, en el tercer baremo Poco Satisfactorio, a los puntajes inferiores de la escala, esto es del 10\% al $40 \%$.

La segunda fase del proyecto fue analizar los factores que inciden en el desempeño académico, el objetivo de está fase consistió en identificar desde la perspectiva de los docentes y estudiantes, cuál es el factor de mayor incidencia que afecta el desempeño de los estudiantes de la muestra bajo estudio, para elaborar un cuadro comparativo que permita contrastar sus percepciones. El enfoque metodológico utilizado en esta fase de la investigación, fue abordado de forma cuantitativa, las encuestas fueron aplicadas con la colaboración de las asesoras pedagógicas de cada una de las nueve Facultades de la UCSG, para el análisis de los datos se utilizaron medidas de tendencia central de la estadística descriptiva, como la mediana y la media.

La información recolectada, fue apoyada en dos cuestionarios con preguntas cerradas y de elección múltiple, donde los encuestados escogieron la respuesta según su criterio o apreciación. Los datos recogidos fueron organizados en una tabla de distribución de frecuencias, la misma que permitió el análisis e interpretación de las características que describen.

La tercera fase de la investigación residió en realizar mejoras al modelo de acompañamiento pedagógico de la UCSG; el objetivo fue rediseñar el modelo de acompañamiento actual, de tal manera que se logre una mayor contribución a mejorar el desempeño de los estudiantes de los tres primeros ciclos, en base a los resultados obtenidos en las fases anteriores y considerando las buenas prácticas de programas similares al nuestro, que han sido documentadas en investigaciones previas de universidades iberoamericanas.

DISCUSIÓN DE RESULTADOS:

Los resultados de la primera variable, se presentan en la siguiente tabla, que sistematiza el resultado del primer objetivo, que consistió en determinar el nivel de efectividad de la Asesoría Pedagógica en las diferentes Facultades de la UCSG, realizando una evaluación de los logros de los estudiantes asesorados, para elaborar un cuadro estadístico. Por lo tanto podemos concluir con esta investigación, que el programa APE en la UCSG, en una escala tipo Likert, alcanzó un nivel de eficiencia Satisfactorio. El nivel de aspiración que persigue la UCSG, para el bienestar de sus estudiantes es elevar la eficacia del programa a Muy Satisfactorio.

Los resultados de la segunda variable se presentan en un cuadro comparativo de Docentes y Estudiantes. En base a los resultados obtenidos se puede interpretar que los porcentajes más altos, tanto en las respuestas de los docentes como en la de los estudiantes, afirman que existe una contraposición en cuanto a las dificultades que 
TABLA 1. CUADRO ESTADÍSTICO PARA EVALUAR EL NIVEL DE EFECTIVIDAD DEL PROGRAMA APE A TRAVÉS DE LOS LOGROS ACADÉMICOS DE LOS ESTUDIANTES ASESORADOS

\begin{tabular}{|c|c|c|c|}
\hline FACULTAD & CARRERA & $\begin{array}{l}\text { PROMEDIO DE } \\
\text { EFECTIVIDAD AÑO } 2015\end{array}$ & $\begin{array}{l}\text { PROMEDIO DE } \\
\text { EFECTIVIDAD AÑO } 2016\end{array}$ \\
\hline \multirow{3}{*}{ Facultad de Arquitectura y Diseño } & Arquitectura & 43,50 & 56,00 \\
\hline & Diseño de Interiores & 54,50 & 45,00 \\
\hline & Gestión Gráfica y Publicitaria & 60,00 & 59,50 \\
\hline \multirow{3}{*}{ Facultad de Artes y Humanidades } & Música & 81,00 & 56,50 \\
\hline & Producción y Artes audiovisuales & 49,50 & 69,50 \\
\hline & Lengua Inglesa & 62,50 & 50,00 \\
\hline \multirow{4}{*}{ Facultad de Ciencias Económicas y Administrativas } & Economía & 60,50 & 50,00 \\
\hline & Gestión Empresarial Internacional & 48,50 & 58,00 \\
\hline & Contabilidad & 45,00 & 63,00 \\
\hline & Administración de Empresas & 56,00 & 56,50 \\
\hline Facultad de Ciencias Médicas & Medicina & 63,39 & 73,22 \\
\hline \multirow{5}{*}{ Facultad de Especialidades Empresariales } & Turismo & 43,00 & 63,50 \\
\hline & Marketing & 86,00 & 67,50 \\
\hline & Comercio y Finanzas Internacionales & 55,00 & 50,50 \\
\hline & Emprendimiento & 78,50 & 54,50 \\
\hline & Ventas & 100,00 & 100,00 \\
\hline \multirow{3}{*}{ Facultad de Filosofía, Letras y Ciencias de la Educación } & Pedagogía & 81,82 & 76,92 \\
\hline & Psicología & 75,90 & 81,93 \\
\hline & Comunicación Social & 71,82 & 62,22 \\
\hline \multirow{2}{*}{ Facultad de Ingeniería } & Ingeniería Civil & 45,50 & 31,00 \\
\hline & Ingeniería en Sistemas Computacionales & 47,50 & 39,50 \\
\hline \multirow{2}{*}{ Facultad de Jurisprudencia y Ciencias Sociales y Políticas } & Derecho & 80,96 & 71,79 \\
\hline & Trabajo Social y Desarrollo Humano & 76,45 & 75,64 \\
\hline \multirow{5}{*}{ Facultad Técnica para el Desarrollo } & Ingeniería Agropecuaria & 41,38 & 54,95 \\
\hline & Medicina Veterinaria & 62,22 & 56,25 \\
\hline & Ingeniería Agroindustrial & 30,00 & 33,33 \\
\hline & Ingeniería en Electro Mecánica & 40,48 & 30,21 \\
\hline & Ingeniería en Telecomunicaciones & 71,67 & 77,52 \\
\hline
\end{tabular}

Nota: Elaboración propia.

A pesar de estar en contraposición vale decir que están estrechamente ligadas, con una flecha en doble vía, ya que el desempeño académico va a estar condicionado al interés por aprender, lo cual va a depender de los recursos metodológipor los docentes.

TABLA 2. CUADRO COMPARATIVO DE ESTUDIANTES Y DOCENTES - \#1

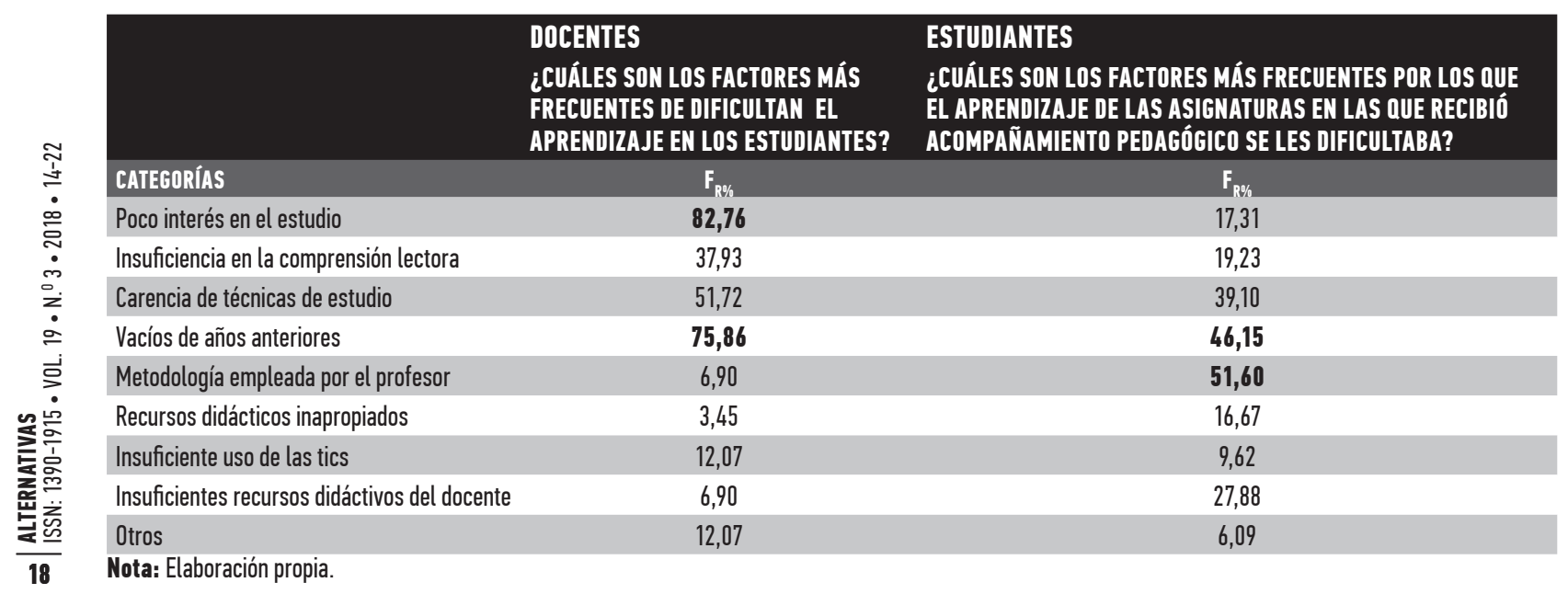


cos empleados por docentes; así también la metodología del docente va a estar condicionada al interés que tenga el estudiante por aprender, ya que aunque el docente sea muy recursivo en cuanto a su metodología a la hora de dictar clases, muchos estudiantes no prestan atención y no le ponen el suficiente interés a la asignatura.

Es interesante también poder apreciar que tanto docentes como estudiantes apuntan al vacío de conocimientos de años anteriores como el motivo que dificulta el aprendizaje, lo cual se encuentra directamente anclado con la metodología usada a la hora de impartir la clase y el poco interés del estudiante dentro del aula, ya que si no se pudo establecer la transferencia docente-estudiante en la materia dictada difícilmente se podrá alcanzar los resultados de aprendizaje previstos.

Cabe recalcar que los estudiantes de la actual generación esperan clases más dinámicas, con metodologías innovadoras, contextualizadas en el marco de una cultura globalizada donde la tecnología sobresale, para atender esta demanda de los estudiantes es necesario que los docentes puedan identificar el estilo de aprendizaje que los alumnos evidencian a la hora de adquirir la información y posteriormente convertirla en aprendizaje efectivo.

El conocimiento sobre los estilos de aprendizaje, le permitirá al docente, planificar su clase utilizando diferentes estrategias metodológicas en su enseñanza y así poder hacer del aprendizaje una experiencia distinta y gratificante en las aulas, en las que los estudiantes se sientan motivados y en confianza para realizar sus actividades educativas, lo que facilita su aprendizaje y su rendimiento. Una buena estrategia es la capacitación del docente en el uso de metodologías activas para mejorar el proceso de enseñanza-aprendizaje y lograr que los estudiantes desarrollen un pensamiento reflexivo, crítico y participativo.

Es así como en el segundo cuadro comparativo, expuesto a continuación, se puede apreciar que la mayoría $(60,34 \%)$ de los docentes emplean como técnica talleres y el $55,17 \%$ el trabajo colaborativo. Aquí es preciso cuestionarse si estas técnicas están siendo llevadas a cabo de forma que permitan un aprendizaje significativo y que permita al estudiante involucrarse en la construcción de su aprendizaje, forjando en él un interés por sus estudios.

En contrastea esto, los resultados dela encuesta aplicada a los estudiantes permite evidenciar que éstos a la hora de ejecutar sus actividades académicas, la mayoría $47,12 \%$ observa videos y el $42,31 \%$ tiende a memorizar lo transmitido por el docente, lo cual se puede interpretar que el estudiante de los primeros ciclos para poder desarrollarse con éxito en sus estudios usan medios virtuales, propios de la era tecnológica en la que crecieron y se desarrollaron, que les permita tener una mayor comprensión de los contenidos impartidos en las diferentes materias de la carrera elegida o simplemente tienden a memorizar contenidos, lo cual está enteramente ligado con el sentir tanto de docentes $(75,86 \%)$ y estudiantes $(46,15 \%)$ de acuerdo a lo evidenciado en la Tabla 1 , al manifestar que las dificultades están en relación a los vacíos académicos de años anteriores y es que una falta de conocimientos previos en el estudiante, le impide relacionar los contenidos nuevos con la información que se halla en su estructura cognitiva.

TABLA 3. CUADRO COMPARATIVO DE ESTUDIANTES Y DOCENTES - \#2

\begin{tabular}{|c|c|c|c|}
\hline \multicolumn{2}{|c|}{$\begin{array}{l}\text { DOCENTES } \\
\text { ¿CUÁLES SON LAS TÉCNICAS QUE USTEd USA PARA DAR CLASES? }\end{array}$} & \multicolumn{2}{|c|}{$\begin{array}{l}\text { ESTUDIANTES } \\
\text { AL EJECUTAR SUS ACTIVIDADES ACADÉMICAS ¿CUÁLES DE LAS SIGUIENTES TÉCNICAS UTILIZA? }\end{array}$} \\
\hline CATEGORÍAS & $\mathbf{F}_{\mathrm{R} \%}$ & CATEGORÍAS & $\mathbf{F}_{\mathrm{R} \%}$ \\
\hline Trabajo Colaborativo & 55,17 & Estudiar para memorizar información & 42,31 \\
\hline Mapas conceptuales y esquemas gráficos & 31,03 & Elaborar organizadores gráficos & 27,88 \\
\hline Estudio de casos & 46,55 & Analizar casos & 23,40 \\
\hline Videos 0 audios & 34,48 & Observar videos & 47,12 \\
\hline Resolución de problemas & 50,00 & Escuchar audios & 18,91 \\
\hline Comprensión lectora & 15,52 & Resolver problemas & 23,40 \\
\hline Resumen de textos & 8,62 & Leer comprensivamente & 37,82 \\
\hline Talleres & 60,34 & Contestar cuestionarios de preguntas & 12,50 \\
\hline \multirow[t]{4}{*}{ Conferencia } & 27,59 & Elaborar resúmenes & 41,03 \\
\hline & & Recibir explicaciones de un compañero & 30,45 \\
\hline & & Estudiar en grupo con compañeros & 31,09 \\
\hline & & Revisar y desarrollar talleres relacionados con la materia & 14,42 \\
\hline
\end{tabular}


TABLA 4. PROPUESTA DE REDISEÑO DEL PROGRAMA DE ASESORÍA PEDAgÓGICA

\begin{tabular}{|c|c|c|}
\hline & $\begin{array}{l}\text { COMPONENTES DEL MODELO DE ACOMPAÑAMIENTO PEDAGÓGICO } \\
\text { ACTUAL }\end{array}$ & COMPONENTES DEL MODELO DE ACOMPAÑAMIENTO PEDAGÓGICO PROPUESTO \\
\hline \multirow{3}{*}{ 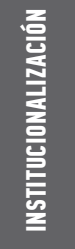 } & Lineamientos no oficializados en la Universidad & Debe funcionar con Lineamientos oficializados en la Universidad. \\
\hline & $\begin{array}{l}\text { Programa APE no está considerado en el Plan Estratégico } \\
\text { Institucional. }\end{array}$ & Debe ser considerado en el Plan Estratégico Institucional \\
\hline & $\begin{array}{l}\text { Se orienta con una visión, misión y objetivos estratégicos no } \\
\text { regulados institucionalmente. }\end{array}$ & $\begin{array}{l}\text { Debe estar orientado con una visión, misión y objetivos estratégicos regulados } \\
\text { institucionalmente. }\end{array}$ \\
\hline \multirow{3}{*}{ 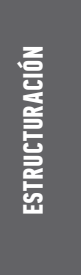 } & Estructura Organizativa no institucionalizada & Debe plantearse una Estructura Organizativa institucionalizada. \\
\hline & $\begin{array}{l}\text { Asesoría Pedagógica Estudiantil sin descripción de cargo y perfil } \\
\text { reconocido institucionalmente }\end{array}$ & $\begin{array}{l}\text { Se plantea una Asesoría Pedagógica Estudiantil con descripción de cargos y } \\
\text { perfiles reconocidos institucionalmente. }\end{array}$ \\
\hline & $\begin{array}{l}\text { Ayudantes Pedagógicos seleccionados por alto rendimiento } \\
\text { académico. }\end{array}$ & $\begin{array}{l}\text { Estudiante Tutor, seleccionado por alto rendimiento académico, por } \\
\text { competencias y con actitud proactiva para el trabajo colaborativo y liderazgo } \\
\text { para el trabajo autónomo de los estudiantes }\end{array}$ \\
\hline \multirow{3}{*}{ 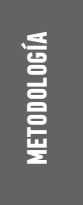 } & $\begin{array}{l}\text { Modelo de acompañamiento pedagógico estrictamente centrado en } \\
\text { el estudiante. }\end{array}$ & $\begin{array}{l}\text { Modelo de acompañamiento pedagógico centrado en el estudiante, estudiante } \\
\text { tutor y el docente. }\end{array}$ \\
\hline & Metodología de pares (Ayudante Pedagógico-estudiante) & $\begin{array}{l}\text { Metodología de pares (Estudiante Tutor- estudiante) con orientación del } \\
\text { docente. }\end{array}$ \\
\hline & Asesoría pedagógica exclusivamente presencial & Asesoría pedagógica presencial y también virtual \\
\hline \multirow{2}{*}{ 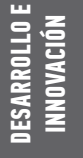 } & $\begin{array}{l}\text { No evalúa estilos de aprendizaje de los estudiantes atendidos por el } \\
\text { programa }\end{array}$ & $\begin{array}{l}\text { Desarrollo de instrumentos para evaluar los estilos de aprendizaje de } \\
\text { estudiantes atendidos por el programa. }\end{array}$ \\
\hline & Capacitaciones no específicas para los requerimientos de APE & $\begin{array}{l}\text { Capacitaciones específicas para los requerimientos de APE en el nuevo } \\
\text { modelo }\end{array}$ \\
\hline \multirow{2}{*}{ 总量 } & Gestión administrativa y académica. & Gestión administrativa: (planificación, ejecución y evaluación). \\
\hline & Evaluación no periódica & $\begin{array}{l}\text { Evaluación periódica: efectividad del programa, eficacia de las estrategias } \\
\text { metodológicas y valoración del desempeño de los miembros de APE. }\end{array}$ \\
\hline
\end{tabular}

Nota: Elaboración propia.

Cabe resaltar que solo un $34,48 \%$ de docentes usa como técnica para dar clases los videos o audios, por medio de lo cual se puede evidenciar la necesidad de que los docentes que en su mayoría, tienen edades entre 36 y 55 años, incluyan en su metodología estos recursos tecnológicos con la finalidad de captar el interés de sus estudiantes, permitiéndoles ser partícipes de su aprendizaje.

Ausubel (1983) sostiene, que existen dos principios de aprendizaje que operan en el aula de clase: el de repetición - aprendizaje significativo y el principio de recepción por descubrimiento, estos principios son válidos siempre que el estudiante relacione el nuevo conocimiento que le facilita el docente con su estructura cognitiva y que el material suministrado sea de interés para él.

Cuando el docente presenta una nueva información a un estudiante que no tiene $\mathrm{co}^{-}$ nocimientos previos, el aprendizaje que se produce es un aprendizaje repetitivo, memorístico que se caracteriza porque no existe ninguna relación entre las experiencias previas del estudiante y ésta, el alumno se ve en la necesidad de memorizarlo porque no alcanza a comprender el significado, la coherencia, la validez y su correspondiente organización interna.

Por lo anteriormente expuesto se puede dimensionar lo importante que es atender este problema y definir una estrategia que permita al estudiante llenar esos vacíos que dificultan su proceso de aprendizaje, pues estos indicadores representan

un riesgo a la hora de formarse como profesionales en las diferentes áreas porque al estudiar una carrera universitaria es imprescindible que el estudiante pueda aprender y aprehender conocimientos que le permitan saber hacer con su profesión en el ámbito laboral. Los resultados de la tercera variable se presentan en el siguiente cuadro que compara el estado del programa actual y las propuestas de reforma.

De acuerdo a investigaciones previas y bibliografías revisadas de los programas de tutoría de pares acerca del acompañamiento pedagógico estudiantil, en Instituciones de Educación Superior en Iberoamérica, los resultados obtenidos, dan cuenta que, todas ejercen sus actividades desde diferentes ámbitos: docencia, académico, gestión administrativa 
y social; están alineadas a un marco jurídico, producto de las últimas reformas educativas y poseen políticas que permitirán que la ciencia avance en los conceptos de tutorías de pares, se ha comprobado de acuerdo a las teorías analizadas, que estas, han mejorado notablemente el rendimiento académico de los estudiantes que participan de estos programas.

El programa de apoyo pedagógico estudiantil, al igual que los programas de otras universidades, tributan a la formación integral de los estudiantes, unas se enfocan al proceso de adaptación e integración del estudiante al sistema de estudio universitario y otras contribuyen a la reducción de la deserción estudiantil y mejorar la tasa de eficiencia terminal de las universidades. APE de la UCSG debe continuar apoyando a la universidad en su estrategia de atención para que en sus estudiantes incrementen su rendimiento académico, es decir sus hábitos de estudio y trabajo colaborativo.

De la misma manera que los programas de tutoría de pares estudiados en otras universidades, APE necesita formalizar la estructura que la rige, esto le permitirá facilitar a los miembros que integran el programa, el conocimiento de su ubicación en la estructura organizacional, establecer la especialización, la departamentalización, la cadena de mando, el tramo de control, la centralización, niveles jerárquicos, identificar relaciones entre los principales órganos de la institución, definir funciones y coordinar la comunicación. Asimismo desde el Vicerrectorado Académico, se deben emitir claramente las políticas, objetivos y las estrategias de acompañamiento que orienten el accionar de APE UCSG.

Se espera que las propuestas planteadas generen una planificación cuidadosa y una formación básica de los estudiantes tutores, la posibilidad de un aprendizaje bidireccional, interactivo, cooperativo; el desarrollo de ciertas habilidades tanto cognitivas como comunicacionales sobre este conjunto de habilidades puestas en juego, desarrollan las competencias cooperativas entre los estudiantes tutores y tutorados, las cuales son transferibles desde el campo educativo y luego al profesional.

\section{CONCLUSIONES}

Una vez concluida la investigación, podemos establecer que el programa APE, de acuerdo a las respuestas obtenidas en las encuestas realizadas a los usuarios en las 9 facultades de la UCSG, logró un Nivel Satisfactorio.
De acuerdo al segundo objetivo planteado, se determina que entre docentes y estudiantes hay un punto de vista en el cual ambos coinciden, los vacíos de conocimientos de años anteriores afectan al proceso de aprendizaje y al desempeño académico.

El tercer objetivo de acuerdo a los resultados de los datos obtenidos en la investigación y a la revisión bibliográfica de las experiencias de universidades Iberoamericanas, donde está implementado con resultados positivos el programa asesoría pedagógica estudiantil; concluimos que la propuesta de institucionalizar el programa APE, es pertinente.

RECOMENDACIONES

De acuerdo a los retos que plantean las últimas reformas en las instituciones de educación superior, la UCSC debe elevar la calidad de la estrategia de acompañamiento estudiantil, al nivel de excelencia académica.

La UCSC debe ofertar cursos propedéuticos para aquellos estudiantes que no alcancen el promedio mínimo requerido, con el fin de nivelarlos y poder cerrar la brecha de los vacíos de conocimiento que les impide construir nuevos aprendizajes.

Institucionalizar el programa de Asesoría Pedagógica Estudiantil, (APE) en la UCSG, para que se convierta en un apoyo y acompañamiento permanente para los estudiantes, siendo un espacio donde las estrategias estén enmarcadas en lo académico dando importancia a todos los estudiantes de grado.

Se propone que esta propuesta vaya al debate de la Comisión Académica de la UCSC para que sea retroalimentada, $y$, si esta reforma es considerada pertinente, pase nuevamente para que sea aprobada por el Consejo Universitario.

El modelo tutorías de pares, de acuerdo con experiencias de otras universidades, debe ser acompañado de un docente tutor.

\section{REFERENCIAS BIBLIOGRÁFICAS}

Álvarez, P. (2014). La función tutorial del profesorado universitario: Una nueva competencia de la labor docente en el contexto del EEES. Revista Portuguesa de Pedagogía, 47(2), 85-106.

Albanaes, P.; Marques de Sousa, F. y Patta Bardagi, M. (2015). Programas de tutoría y mentoría en universidades brasileñas: Un estudio bibliométrico. Revista de Psicología, 33(1), 21-56.

Ausubel, D.P. (1976).Psicología educativa: un punto de vista cognoscitivo. México, Editorial Trillas. p. 58.

Bianculli, K. y Marchal, M. (2013). Las tutorías universitarias: estudio de caso:Programa de Tutorías PACE- 
NI de la Facultad de Ciencias Económicas y Sociales de la UNMdP. Mar del Plata: Universidad Nacional de Mar del Plata.

Casado-Muñoz, R.; Lezcano-Barbero, F. y Colomer-Feliu, J. (2015). Diez pasos clave en el desarrollo de un programa de mentoría universitaria para estudiantes de nuevo ingreso. Revista Electrónica Educare [en línea], 19(2), 155-179. <http://www.una.ac.cr/ educare>.

Durán, D. (2009). Aprender a cooperar. Del grupo al equipo. En J.I. Pozo y M.P. Pérez (Eds.), La Psicología del aprendizaje universitario: la formación en competencias. Madrid: Ediciones Morata.

Durán, D. Flores Coll M. (2014). Prácticas de tutoría entre iguales en universidades del Estado español y de Iberoamérica. Revista Iberoamericana sobre $\mathrm{Ca}-$ lidad, Eficacia y Cambio en Educación, REICE. Universitat Autónoma de Barcelona. ISSN: 1696-4713 www.rinace.net/reice/.

Lobato Fraile, C. Nagore Guerra, B. (2016). La tutoría en la educación superior en Iberoamérica: Avances y desafíos. EDUCAR, vol. 52, núm. 2, 2016, pp. 379-398 Universitat Autònoma de Barcelona. Barcelona, España. Disponible en: http://www.redalyc.org/articulo. oa?id=342146063009

Sánchez Ávila, C.; Macías, J.; Almendra, A. y Jiménez Leube, F. J. (2007). Origen, desarrollo, evolución y resultados del Proyecto Mentor en la ETSI de Telecomunicaciones de la Universidad Politécnica de Madrid. Cuaderno de Innovación Educativa en las Enseñanzas Técnicas Universitarias, 1(2), 35-46. 